\title{
Clinical Analysis of Lobular Keloid after Ear Piercing
}

\author{
Hyung Do Kim', \\ Sung Chul Chu', \\ So Min Hwang ${ }^{1}$, \\ Hook Sun ${ }^{2}$, \\ Min Kyu Hwang ${ }^{1}$, \\ Min Wook Kim \\ Jong Seo Lee ${ }^{1}$ \\ ${ }^{1}$ Aesthetic, Plastic and Reconstructive Surgery \\ Center, Good Moonhwa Hospital, Busan; \\ ${ }^{2}$ Department of Plastic and Reconstructive \\ Surgery, Baik Hospital, Busan Inje University, \\ Inje university School of Medicine, Busan, \\ Korea
}

No potential conflict of interest relevant to this article was reported.

\begin{abstract}
Background: Lobular keloid appears to be a consequence of hypertrophic inflammation secondary to ear piercings performed under unsterile conditions. We wish to understand the pathogenesis of lobular keloids and report operative outcomes with a literature review.

Methods: A retrospective review identified 40 cases of lobular keloids between January, 2005 and December, 2010. Patient records were reviewed for preclinical factors such as presence of inflammation after ear piercing prior to keloid development, surgical management, and histopathologic correlation to recurrence.

Results: The operation had been performed by surgical core extirpation or simple excision, postoperative lobular compression, and scar ointments. Perivascular infiltration was noted in intra- and extra-keloid tissue in $70 \%$ of patients. The postoperative recurrence rate was $10 \%$, and most of the patients satisfied with treatment outcomes.

Conclusion: Histological perivascular inflammation is a prominent feature of lobular keloids. Proper surgical treatment, adjuvant treatments, and persistent follow-up observation were sufficient in maintaining a relatively low rates of recurrence.
\end{abstract}

Keywords: Keloid / Inflammatory / Biopsy

\section{INTRODUCTION}

Keloid is a scar tissue that grows over the sites of a previous injury, characterized by excessive deposition of collagen in the dermis during the wound healing process [1]. It commonly occurs in anterior chest, shoulder, upper extremities, and the earlobes [2]. Keloid is more frequently in non-Caucasian populations, particularly the black populations, and is associated with genetic predisposition with certain families having strong history [3]. Although Keloid may occur in all age groups, it is more prevalently in patients between 10 and 30 years of age [4].

Of all types of keloid, lobular keloid occurs has the highest incidence. Lobular keloid has been reported to occur as a secondary

Correspondence: Hook Sun

Department of Plastic and Reconstructive Surgery, Baik Hospital, Busan Inje University, Inje university School of Medicine, 75 Bokji-ro, Busanjin-gu, Busan 47392, Korea

E-mail: sun443@naver.com

Received February 12, 2015 / Revised April 22, 2015 / Accepted December 3, 2015 inflammatory response in patients who have undergone ear piercing at non-medical institutions such as jewelry shop, at home, or school with a piercing gun, needle, and earrings. These types of keloids are managed with a wide variety of treatment methods with good outcomes. However, there are no established methods for effectively predicting or preventing recurrence of lobular keloid [5]. Prevention or treatment methods for lobular keloid include intralesional steroid injection, radiotherapy, compression therapy, surgical excision, silicone sheet application, laser therapy, cryotherapy, interferon- $\alpha$ injection, and pharmacological treatments [6-9].

Given this background, we conducted this study to determine if histopathologic findings of lobular keloidsis associated with recurrence and to evaluate outcomes following a combination therapy consisting of surgical excision, compression therapy with magnetic compression earrings, and scar ointment. 


\section{METHODS}

A single-institution retrospective review identified a total of $36 \mathrm{pa}-$ tients (40 earlobes) who had a history of receiving ear piercing at non-medical (not certified for piercing procedure) and was treated for lobular keloids between January of 2005 and December of 2010.

We reviewed medical records for pertinent clinical interview and physical examination data. Patients were asked to fill out a clinical questionnaire regarding piercing sites, the types of piercing, physical changes following piercing, and the presence of inflammation. In addition, patients were also asked to record sites of keloid, changes in the keloid size, and number of keloids. We also reviewed the method of ear piercing, age at first piercing, age at onset of keloids, and past medical history.

At our institution, surgical modality is chosen depending on whether the keloid distorted the earlobe margin. If the keloid did not extend laterally beyond the site of piercing, the lesion was extirpated as a core with a slight margin. If the keloid did extend laterally beyond the piercing site and extended into the earlobe margin, then the lesion was excised. Intraoperative notes were reviewd to obtain the type of operation patient underwent. Additionally, clinic notes were reviewed for recurrence and ongoing signs of inflammation following keloid excision, as well as histopathologic characteristics in relation to recurrence.

\section{RESULTS}

Almost all of the patients (97\%) were less than 40 years of age. In all 40 cases of lobular keloids, there was no discrepancy between the clinical diagnosis prior to the operation and the postoperative histologic diagnosis. Unlike other types of keloid, inflammatory cells were observed in lobular keloids and adjacent tissue (Fig. 1). Based on the presence of inflammatory cells, the cases were divided into two groups. The first group consisted of those keloids, which demonstrated perivascular inflammation both within the keloid tissue and in the surrounding non-keloid tissue. The second group consisted of those cases in which perivascular inflammation was confined to outside of the keloid tissue itself (Fig. 2). Out of the 40 cases, we found that 28 lobular keloids (70\%) had perivascular inflammatory cell infiltration in both keloid and adjacent areas, with the remaining 12 cases (30\%) having inflammation outside the keloid tissue (Table 1). The frequency of perivascular inflammatory cell infiltration in both intra-keloid and extrakeloid was relatively in most of the recurred cases and the duration of inflammatory responses was relatively longer.

Simple excision was performed for 20 patients, and core exci-

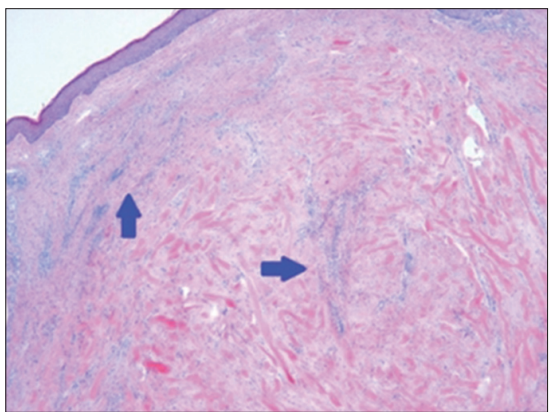

Fig. 1. Histopathologic photograph showing a typical keloid fibers with perivascular inflammatory cell infiltration (blue arrows) $(\mathrm{H} \& \mathrm{E}, \times 100)$.
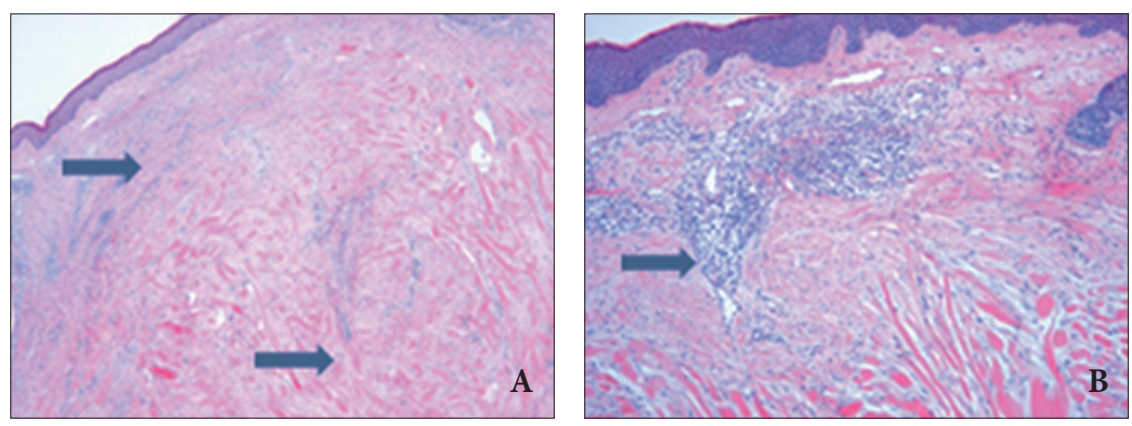

Fig. 2. Histopathologic photographs. (A) Histopathologic photograph showing the perivascular inflammatory cell Infiltration (blue arrows) at the center \& peripheral of keloid (H\&E, $\times 100)$. (B) Histopathologic photograph showing the perivascular inflammatory cell infiltration (blue arrow) at the peripheral of keloid only $(\mathrm{H} \& \mathrm{E}, \times 100)$. 
sion was performed for 16 patients (Fig. 3). Patients were recommended to wear magnetic compression earrings beginning at 2 weeks and to continue for six months after the operation. Scar ointment (Cica 20 ointment, Laboratories D’Armor, Pleubian, France) was also recommended.

Notable induration or erythema at the postoperative site was also evaluated as a potential predictor of recurrence. Out of the 40 cases, 4 lobular keloids had recurred (10\%). One of these patients experienced repeated incidence of recurrence with pregnancy, and this was assumed to be under hormonal influence. The remaining three cases were assumed to occur due to the ongoing inflammation in the postoperative period. The majority of patients were satisfied with treatment outcomes.

\section{DISCUSSION}

The etiologic factors of keloids include skin injuries (surgery, lac-

Table 1. Two types of perivascular inflammatory cell infiltration were found on histopathologic study

\begin{tabular}{ll}
\multicolumn{2}{c}{ Perivascular inflammatory cell infiltration } \\
\hline Intra- \& extrakeloid & Extrakeloid \\
\hline Keloid (mass) and peripheral inflammation & Peripheral inflammation only \\
\hline
\end{tabular}

eration, tattoo, burn injury, injections, and preventive vaccinations), excessive skin tension, and hormonal factors [6]. Of these, the lobular keloid has been suggested to occur secondary to inflammation after ear piercing.

In this current study, all patients had a past history of undergoing ear piercing at non-medical institutions such as jewelry shop, home, or at school. All of the patients had reported a presence of inflammation or abscess after ear piercing but prior to keloid formation. As for sites of keloid formation, the left-side was more prevalent. Most cases of keloid began to develop 1-6 months after inflammatory reaction began to subside and continued to grow for $1-2$ years thereafter (Table 2). The mean keloid size was $11 \times 20 \times$ $15 \mathrm{~mm}$ (range, $2 \times 2 \times 3$ to $40 \times 25 \times 15 \mathrm{~mm}$ ). We found no relationship between keloid size and recurrence.

Our study results suggest that the etiologic factors of lobular keloid include inflammations of the tissue secondary to non-sterile ear piercing environment. Typically, inflammatory cells are not predominant features of keloid histology. However, we have found that lobular keloid was associated with perivascular infiltration of inflammatory cells. Analyzing the pattern of perivascular inflammatory cell infiltration, we found that patients with longer periods of keloid growth ( $>1$ year) tended to have histologic evidence of perivascular inflammation which involved both intra-keloid and
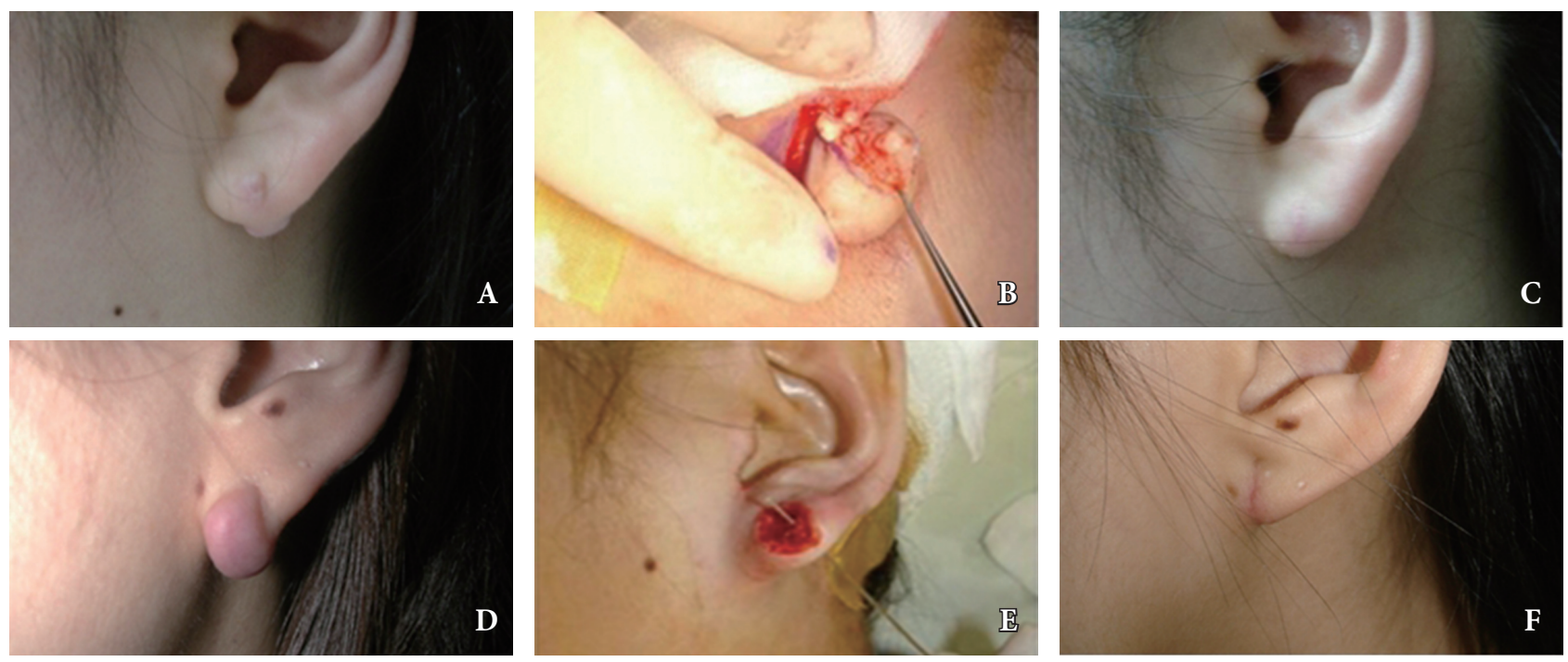

Fig. 3. A 15-year-old female with lobular keloid. (A) Preoperative photograph. (B) Intraoperative photograph (simple surgical excision). (C) Postoperative photograph after 5 months. (D) Preoperative photograph of a 26-year-old female with lobular keloid. (E) Intraoperative photograph (core extirpation). (F) Postoperative photograph after 2 months. 
Table 2. Summary of survey results

\begin{tabular}{lc}
\multicolumn{1}{c}{ Variable } & Results ( 36 patients) \\
\hline Piercing at illegal place (yes/no) & $36 / 0$ \\
Age at piercing & \\
11-18 years old & 11 \\
Over 19 years old & 25 \\
\hline Inflammation (yes/no) & $36 / 0$ \\
Side of keloids (left/right/both) & $24 / 12 / 4$ \\
Duration of keloid growth & \\
$<1$ yr & 15 \\
$\geq 1 \mathrm{yr}$ & 21 \\
Interval between mature keloid to the operation & \\
$<1 \mathrm{yr}$ & 16 \\
$\geq 1 \mathrm{yr}$ & 15 \\
Patients satisfaction & \\
Excellent & 16 \\
Good & 20 \\
\hline
\end{tabular}

extra-keloid tissue. This suggests a higher possibility of perivascular inflammatory cell infiltration in both an intra-keloid and an extra-keloid if the inflammation is of severe degree and lasts for longer periods of time.

Among the four cases of recurrence, one patient reported temporal association between repeated keloid recurrences to each of her pregnancy. The keloid specimen from this patient had perivascular inflammation that was confined to extra-keloid tissue. This was in contrast to remaining three patients in whom perivascular inflammation was found in both intra- and extra-keloid locations. This difference may be a demonstrable difference between hormonal and purely inflammatory stimulation of myofibroblasts, though this would require further basic science and clinical research [10].

Many methods have been attempted for treatment of keloid [11]. However, most of these methods have been effective only in a limited scope. Simple excision has been reported to have a higher rate of recurrence, ranging from $40 \%$ to $100 \%$ [12]. Because of this, surgical excisions are combined with adjunct therapies such as steroid, X-ray and compressive therapy [13].

Regarding the treatment methods for keloid, compression therapy was first reported by Rayer in 1894 . Thereafter, many authors have agreed on its effectiveness. Little is known about the mechanisms by which compressive therapy treats and prevents the formation of keloids. The current standing theory is alterations in col- lagen metabolism due to the compression. Continous compression is suspected to produce local tissue hypoxia, which leads to degeneration of fibroblasts and degradation of collagen fibers [14]. The treatment causes no significant cosmetic problems and causes little or no side effects of steroid injections. One significant disadvantage of compression therapy is the need for long treatment duration.

Based on the histopathologic findings in our study, we suspect inflammation is perhaps a significant contributor to lobular keloids. We also were able to obtain satisfactory treatment outcomes through adjuvant therapies and continuous monitoring of the clinical course. Further studies are warranted to clarify the accurate pathophysiology of lobular keloid.

\section{REFERENCES}

1. Murray JC. Scars and keloids. Dermatol Clin 1993;11:697-708.

2. Burd A, Huang L. Hypertrophic response and keloid diathesis: two very different forms of scar. Plast Reconstr Surg 2005;116:150e-157e.

3. Oluwasanmi JO. Keloids in the African. Clin Plast Surg 1974;1:179-95.

4. Ketchum LD. Hypertrophic scars and keloids. Clin Plast Surg 1977;4:301-10.

5. Mutalik S. Treatment of keloids and hypertrophic scars. Indian J Dermatol Venereol Leprol 2005;71:3-8.

6. Al-Attar A, Mess S, Thomassen JM, Kauffman CL, Davison SP. Keloid pathogenesis and treatment. Plast Reconstr Surg 2006;117:286-300.

7. Park CH, Hong SJ, Chang KH, Jung KN, Kwon TK, Lee JH. Combination therapy of earlobe keloids. Korean J Otolaryngol-Head Neck Surg 2006;49:968-72.

8. Kelly AP. Medical and surgical therapies for keloids. Dermatol Ther 2004;17:212-8.

9. Contin LA, Bastazini I, Alves CJ, do Nascimento DB. Keloids in the ears: follow-up of 41 patients who had surgery and intralesional corticosteroid injections. Surg Cosmet Dermatol 2011;3:109-11.

10. Moustafa MF, Abdel-Fattah MA, Abdel-Fattah DC. Presumptive evidence of the effect of pregnancy estrogens on keloid growth: case report. Plast Reconstr Surg 1975;56:450-3.

11. Atiyeh BS, Costagliola M, Hayek SN. Keloid or hypertrophic scar: the controversy: review of the literature. Ann Plast Surg 2005;54:676-80.

12. Careta MF, Fortes AC, Messina MC, Maruta CW. Combined treatment of earlobe keloids with shaving, cryosurgery, and intralesional steroid injection: a 1-year follow-up. Dermatol Surg 2013;39:734-8.

13. Snyder GB. Button compression for keloids of the lobule. Br J Plast Surg 1974;27:186-7.

14. Kischer CW, Shetlar MR, Shetlar CL. Alteration of hypertrophic scars induced by mechanical pressure. Arch Dermatol 1975;111:60-4. 